\title{
Proceedings of the Second Regional Neurocritical Care Meeting in the Middle East and Africa in conjunction with the 17th Emirates Critical Care Conference (ECCC)
}

\section{(C) 2021 Springer Science+Business Media, LLC, part of Springer Nature and Neurocritical Care Society}

\author{
June 18-19, 2021 - Hybrid Meeting \\ Dubai Festival City \\ Dubai, United Arab Emirates
}

\section{The Review Process}

Yasser B. Abulhasan

Abstracts submitted to the Second Regional Neurocritical Care Meeting in the Middle East and Africa in conjunction with the 17th Emirates Critical Care Conference (ECCC) were assessed by reviewers who were blinded to avoid bias. Raters were not aware of the submitting author(s) or affiliated institution(s). Reviewers graded each abstract using an integer scale from 1 to 7 (1 equals poor and 7 equals outstanding) using forms and guidelines developed by the Neurocritical Care Society. The following criteria were individually assessed: (1) importance and relevance to the field of neurocritical care, (2) originality and innovation, and (3) quality of methods and writing.

I would like to acknowledge the voluntary efforts of this group of dedicated multidisciplinary reviewers and sincerely thank them for their contribution in rating abstracts for the First [1] and Second Regional Meetings:

- Mary Kay Bader

- Gretchen Brophy

- Jose Suarez

- Michel Torbey

- Katja Wartenberg

This meeting was initially scheduled to be in person on April 2-4, 2020, in Dubai. However, it got postponed to June 18-19, 2021 (in a hybrid format [in person and virtual]) because of the COVID-19 global pandemic, associated safety concerns, and international travel restrictions. Exceptionally, the abstract submission was open twice, in 2019-2020 and 2021. Abstracts submitted and accepted in 2020 that got published in full in peerreviewed journals were withdrawn from this publication because of copyright reasons. In those cases, submitting authors were informed of this decision and invited to present their work during the conference.

\section{References}

1. Abstracts Presented at the First Regional Neurocritical Care Meeting in the Middle East and Africa in conjunction with the 15th Emirates Critical Care Conference (ECCC). Neurocritical Care. 2019;30(3):690-5.

\section{Risk of cardiovascular comorbidities following extra-axial TBI: two-institution clinical experience over 30 years}

Ali Al Jarrah, Zabreen Tahir, Taha Yahya, Alexander Brown Whalen, Gallen V. Henderson, and Saef Izzy

Department of Neurology, Brigham and Women's Hospital and Harvard Medical School, Boston, MA, USA

\section{Introduction}

Traumatic brain injury (TBI) is a significant public health problem because of its prevalence and socioeconomic burdens. Studies showed that patients with TBI are at high risk for long-term cardiovascular and cerebrovascular comorbidities after injury; however, little is known about the epidemiology and time line of these comorbidities after different subtypes of TBI. This study 
aimed to assess the risk of selected medical comorbidities and their time of onset following extra-axial TBI.

\section{Methods}

Institutional databases were surveyed to identify adult patients admitted with subdural hematoma (SDH), epidural hematoma $(E D H)$, and traumatic subarachnoid hemorrhage (tSAH) between 1990 and 2020. TBI and comorbidities, including hypertension (HTN), diabetes, acute coronary syndrome, and stroke, were defined by International Classification of Diseases diagnosis codes. Patients with any preexisting comorbidities before TBI were excluded. Patients with extra-axial TBI were identified and followed up over 10 years.

\section{Results}

A total of 33,173 patients $(17,876$ with SDH, 12,322 with tSAH, and 2,975 with EDH) were identified. Stroke was the most common comorbidity in patients with TBI (28\% with SDH, 31\% with tSAH, and $23.1 \%$ with $\mathrm{EDH}$ ), with a short median time to onset of 7 months post tSAH, 10 months post SDH, and 14 months post EDH. HTN was the second most common comorbidity (23\% with $\mathrm{SDH}, 21 \%$ with $\mathrm{tSAH}$, and $23 \%$ with $\mathrm{EDH}$ ), with time to onset of 2.4 years post $\mathrm{tSAH}, 2.8$ years post $\mathrm{SDH}$, and 3.7 years post EDH. After SDH, stroke and acute coronary syndrome were more common in patients older than 60 years, whereas HTN and diabetes were more common in patients younger than 60 years $(p<0.0001)$. After tSAH, both stroke and HTN were more common in patients older than 60 years; however, after EDH, all selected comorbidities were more common in patients older than 60 years compared with patients younger than 60 years $(p<0.0001)$.

\section{Conclusions}

Patients with extra-axial TBI are at potential risk of developing cardiovascular comorbidities post injury. We hope these results will remind critical care physicians and neurosurgeons to keep in mind the long-term effects of extra-axial TBI.

\section{Admission Modified Early Warning Score in neurosurgical patients predicted ICU mortality Babatunde Osinaike and Arinola Sanusi Department of Anaesthesia, University of Ibadan and University College Hospital, Ibadan, Nigeria}

\section{Introduction}

Following surgery or trauma, neurosurgical patients are often monitored in an intensive care setting for a period of time. Many scores have been developed to predict outcomes. In low- and middle-income countries, the applicability of scoring systems is precluded by the unavailability of resources to compile all the parameters of these scores. The aim of this study was to determine the role of the Modified Early Warning Score (MEWS) as a predictor of intensive care unit (ICU) mortality in neurosurgical patients.

\section{Methods}

A retrospective study was conducted on adult patients with neurosurgical diagnoses over 1 year. Patient demographics, admission characteristics, details of neurosurgical diagnoses, ICU interventions, and outcomes were obtained from the ICU record. Univariate and logistic regression analyses were done to determine predictors of ICU nonsurvival. The significance level was set at $p<0.05$.

\section{Results}

Of 286 patients admitted in the study year, 83 had neurosurgical indications. Univariate analysis showed significant differences between the survivor and nonsurvivor group regarding Glasgow Coma Scale on admission $(p=0.003)$, the MEWS on admission $(p=0.04)$, ventilator therapy $(p=0.000)$, vasopressor use $(p=0.001)$, the median daily fluid balance $(p=0.004)$, and ventilator days $(p=0.003)$. Although poorer outcome was observed in the severe head injury, traumatic myelopathy, and clot evacuation groups, the difference was not statistically significant $(p=0.34)$. Following logistic regression, only the MEWS on admission predicted poor outcome $(p=0.04$, $\beta=1.63$ ).

\section{Conclusions}

A higher MEWS on admission predicted poor outcome. With the increasing burden from complications of critical illness in resource-challenged settings, particularly in sub-Saharan Africa, the MEWS may be a useful scoring system for in-hospital mortality prediction. However, large prospective studies are needed.

\author{
Incidence of dementia after acute brain injury: \\ two-institution clinical experience over 15 years \\ Zabreen Tahir*, Farid Radmanesh*, Taha Yahya, Ali Al \\ Jarrah, Winnie Li, Hadi Abou El Hassan, Samuel Snider, \\ Galen V. Henderson, and Saef Izzy \\ "Equally contributed \\ Departments of Neurology and Neurosurgery, Brigham \\ and Women's Hospital and Harvard Medical School, Boston, \\ MA, USA
}




\section{Introduction}

Survivors of intracerebral hemorrhage $(\mathrm{ICH})$ are at increased risk of cognitive decline. We hypothesize that the risk of dementia differs in traumatic compared with spontaneous ICH. In this study, we compared the risk of dementia in young patients with traumatic versus spontaneous $\mathrm{ICH}$.

\section{Methods}

We conducted a retrospective cohort study (2000-2018) of patients with ICH between the age of 20 and 60 years who were observed for 10 years. Traumatic and spontaneous ICH and other phenotypes were determined using International Classification of Diseases codes. Patients with preexisting dementia or those diagnosed with dementia within 6 months after ICH were excluded. Cox proportional hazards and logistic regression models were used to assess the risk and predictors of dementia, respectively.

\section{Results}

Of 8,733 patients with $\mathrm{ICH}, 2,995$ (34\%) had traumatic $\mathrm{ICH}$ and 5,738 (66\%) had spontaneous ICH. Patients with traumatic ICH were younger (median 46 years [interquartile range 34-54]; $p<0.001$ ) than patients with spontaneous ICH (median 49 years [interquartile range 41-55]). The 10-year cumulative incidence of dementia after spontaneous $\mathrm{ICH}$ was $1.3 \%$, compared to $2.1 \%$ after traumatic ICH $(p=0.002)$. There was no significant difference in the time to dementia between the two groups $(p=0.47)$. The risk of dementia after traumatic ICH was higher than that after spontaneous ICH (hazard ratio [HR] 1.5 [95\% confidence interval $\{\mathrm{CI}\} 1.1-2.2]$; $p=0.02$ ). The predictors of dementia, by using multivariable logistic regression, include thyroid disorders (HR 2.7 [95\% CI 1.2-5.5]), depression (HR 1.6 [95\% CI 1.1-2.4]), and alcohol misuse (HR 1.8 [95\% CI 1.1-2.7]).

\section{Conclusions}

Patients with $\mathrm{ICH}$, particularly those secondary to brain trauma, are at increased risk of cognitive decline at a relatively young age. These data highlight the long-term complications of acute brain injuries and the importance of preventative measures and risk reduction strategies.

\section{Nurses' perceived barriers to early mobilization in a neuroscience critical care unit: tertiary hospital in Abu Dhabi \\ Nimeh Omar Mahmoud \\ Neuro-ICU, Cleveland Clinic Abu Dhabi, Abu Dhabi, United Arab Emirates}

\section{Introduction}

Patients admitted to the critical care unit often face several debilitating outcomes, including neuromuscular dysfunction (which was previously reported among more than $40 \%$ of the patients after an extended use of mechanical ventilation) and longer stays without mobilization. As such, this quantitative study sought to determine some of the leading barriers to early mobilization of patients within the neuroscience critical care unit (NCCU), as perceived by the nurses.

\section{Methods}

The researcher randomly recruited a sample of 46 nurses who operate within the NCCU of the tertiary care hospital in Abu Dhabi before administering the research questionnaire. A borrowed and validated questionnaire was used to collect the participants' responses in terms of attitude, knowledge, and behaviors toward early mobilization in the NCCU. The resultant responses were thus statistically analyzed using the analytical tools of the IBM SPSS statistical package.

\section{Results}

The total knowledge mean score was 16.29 (SD 1.75), showing that the claims of possessing high knowledge about patient's mobility was high among the nurses of the studied sample. An attitude total score of 29.70 (SD 4.07) was an indicator of moderate attitude toward the mobility of the patients within the NCCU. Lastly, a moderate behavior score of 42.96 (SD 6.00) was recorded among the participants. Further analysis showed a statistically significant positive correlation between the three variables, knowledge $(p=0.003)$, attitude $(p=0.000)$, and behavior $(p=0.000)$, and a high Pearson's correlation coefficients $(0.515,0.784$ and 0.885 , respectively). The overall questionnaire had acceptable reliability (Cronbach's alpha of $0.768[0.7 \leq \alpha<0.8])$.

\section{Conclusions}

The findings established by this quantitative data analysis conclude that in the NCCU, nurses are averagely equipped with the necessary skills needed for early patient mobilization. However, such skills are not perfectly exhibited among the participants, and any negative fluctuation can critically hamper the practice of early patient mobilization. Moreover, this research has shown that issues of knowledge, attitude, and behavior play a vital role in early patient mobilization. 
External ventricular drains

versus intraparenchymal pressure monitors in the management of severe traumatic brain injury: experience at two academic centers over a decade Zabreen Tahir, Brittany Stopa, Ali Al Jarrah, Taha Yahya, Saef Izzy, and William Gormley Departments of Neurology and Neurosurgery, Brigham and Women's Hospital and Harvard Medical School, Boston, MA, USA

\section{Introduction}

Severe traumatic brain injury (sTBI) is a major cause of mortality and long-term disability. Although intracranial pressure (ICP) monitoring is recommended in sTBI management, the choice between the external ventricular drain (EVD) and intraparenchymal monitor (IPM) is still controversial, and their correlation with long-term post-traumatic-brain-injury outcome is largely unknown. This study aimed to assess the impact of the ICP monitoring type on discharge outcomes and 30-day mortality of patients with sTBI.

\section{Methods}

Institutional databases were surveyed to identify patients with sTBI between 2007 and 2017. sTBI was defined as the presence of an ICP monitor, ventilatory assistance for more than 24 consecutive hours, and International Classification of Diseases, Ninth Revision/International Classification of Diseases, Tenth Revision traumatic brain injury diagnosis codes. Hospital length of stay, the outcome at discharge, and 30-day mortality were evaluated between patients monitored with EVD and those monitored with IPM.

\section{Results}

A total of 589 patients with sTBI were identified; $38.4 \%$ were monitored with EVD, and $61.6 \%$ were monitored with IPM. Mean age $(46.1 \pm 19.5$ vs. $48.3 \pm 20.2)$ and sex were comparable between the two groups. The EVD group had more intraparenchymal hemorrhages and midline shift (MLS) compared with the IPM group $(p=0.03)$. The EVD group required more hyperosmolar therapy compared with IPM group (63.3\% vs. $36.4 \%$; $p<0.001)$. There was no difference in the craniotomy rate between the groups. The EVD group had significantly longer hospital length of stay compared with the IPM group ( 21 vs. 5 days; $p<0.001$ ). Discharge to long-term care or a rehabilitation facility did not vary between the groups (67.3\% of EVD group vs. 59.5\% of IPM group). There was no difference in 30-day mortality between the groups (EVD 24.3\% vs. IPM 29.5\%). Older age, lower motor Glasgow Coma Scale score, and MLS were associated 30-day mortality $(p<0.001)$.

\section{Conclusions}

Patients with sTBI requiring EVD monitoring had more intraparenchymal hemorrhages and MLS and required more hyperosmolar therapy. However, the discharge disposition and 30-day mortality outcomes were comparable between patients monitored with EVD and those monitored with IPM. Future prospective studies to evaluate the long-term outcome of ICP targeted management are still warranted.

\section{Publisher's Note}

Springer Nature remains neutral with regard to jurisdictional claims in published maps and institutional affiliations. 